\title{
Capacity Configuration and Selection of Emergency Power Supply for Wind Farms under Typhoon Conditions
}

\author{
Zhongwen Qian ${ }^{1}$, Qian Guo ${ }^{2}$, Qi Zhang ${ }^{3}$, Dan Zhou ${ }^{3 *}$, Songlie Zhao ${ }^{2}$ and Luyao Xie ${ }^{3}$ \\ ${ }^{1}$ Zhejiang Provincial Energy Group Company, Ltd, Hangzhou, Zhejiang, 310000, China \\ ${ }^{2}$ Guodian Power Zhejiang Zhoushan Offshore Wind Power Development Co., Ltd, Zhoushan, Zhejiang, 316000, China \\ ${ }^{3}$ College of Information Engineering, Zhejiang University of Technology, Hangzhou, Zhejiang, 310014, China
}

\begin{abstract}
China's coastal areas are rich in wind energy resources, and there is a huge market demand for wind power generators. However, due to the impact of typhoons every summer, the wind farms are cut off, causing serious damage to the wind turbines and causing significant economic losses. Based on optimizing the yaw system under typhoon conditions, this paper configures and selects the capacity of diesel generators. First, this article introduces the structure and main functions of the yaw control system. Secondly, through the study of the under-stage wind field model, the algorithm of wind speed extreme value and the calculation method of yaw angle and yaw direction are proposed, and hill climbing algorithm is introduced to optimize the work of the yaw control system. Then, a method for calculating the actual power of a single yaw control system is introduced. On this basis, the capacity configuration plan of the standby diesel generator set was determined, so that it can meet the normal yaw work of the whole field yaw system in the event of a power outage in the field to ensure the safety of the wind turbine.
\end{abstract}

\section{Introduction}

The coastal areas are rich in wind energy resources, but the operation and maintenance of wind farms are very difficult due to the frequent typhoons. If the wind farm is in the state of power failure, how to ensure the reliable operation of the yaw system of wind turbines in coastal areas, realize the protection of wind turbines, and thus reduce the economic loss of wind farms is the key to the construction of wind farms in coastal areas under extreme working conditions.

Only some domestic wind farms use diesel generators as emergency backup power. In literature [1], in order to study the dynamic characteristics and failure mechanism of wind turbines in the case of typhoons, the coordinated superposition method is adopted to calculate the fluctuation time history of typhoons, and the load profile of wind turbines is simulated through simulation. Literature [2] adopts time domain simulation method to estimate the inrush current of the wind generator transformer during the isolated island operation of a large offshore wind farm, so as to allocate auxiliary diesel driven synchronous generator to provide power for the whole wind farm. In literature [3], an orderly wind abandon strategy for offshore wind power with grid elasticity in mind is proposed, which can effectively make use of the regulatory capacity of the system and avoid the impact of short wind power slope events and severe shortage on the balance of supply and demand. In literature [4], by analyzing the failure principle of wind turbines under typhoon conditions, it is concluded that the adoption of force reduction system in terms of mechanics can improve the load capacity of wind turbines to some extent. Literature [5] first estimated the load of wind turbines under typhoons, and on this basis adopted the normal pitch - flying plume grounding strategy and wind turbine stopping strategy to ensure the safety of blade structure.

In summary, the existing literature only estimates the damage of typhoon conditions, and only adopts the method of mechanical reinforcement for prevention and control measures, without considering the damage of wind turbine generators through yaw system. To this end, this paper firstly optimizes the yaw system of wind turbines, realizes the optimal control of yaw system in the face of different wind direction and wind speed, and then realizes the optimal type and capacity configuration of diesel generator set according to the optimized yaw system.

\section{Yaw system control strategy}

\subsection{Overview of yaw control system}

The principle diagram of yaw control system is shown in Figure 1. 


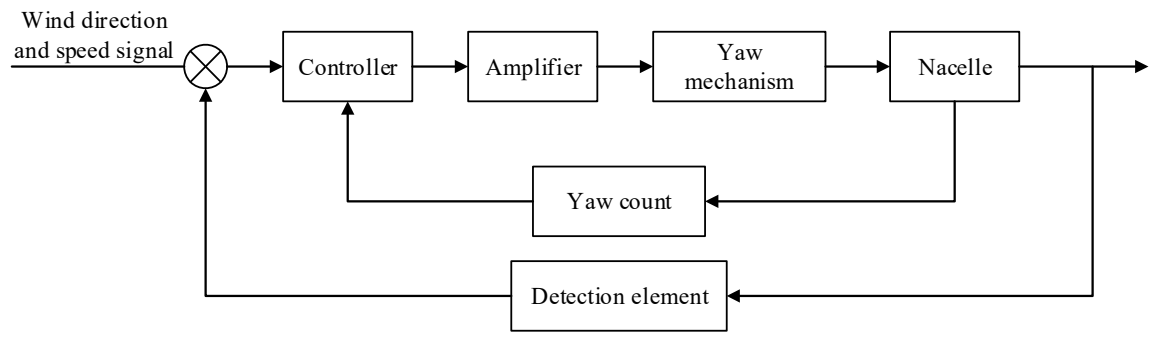

Figure 1. Block diagram of yaw control system

\subsection{Optimization strategy of yaw control system}

In this paper, in order to ensure that the wind turbine is not damaged under typhoon conditions, the minimum output value of the wind turbine is calculated based on the improved mountain-climbing algorithm, so as to minimize the load borne by the wind turbine. First, assume that the wind speed is unchanged. When the wind direction changes, the output power of wind turbine will change. Set the current output power value of the fan as $P_{\text {new }}=P_{1}$ and compare it with the minimum output power value $P_{\text {min }}$ in the current operation curve of the wind turbine. If it is $\left|P_{\text {new }}-P_{\text {min }}\right|>P_{e 1}$, the yaw motor will be started for yaw, and the yaw will be carried out clockwise according to the initial deflection direction set in advance. After yaw once, obtain the output power of the new cycle again, which is $P_{\text {new }}=P_{2}$, and assign the power $P_{1}$ at the previous moment to $P_{\text {old }}$, which is $P_{\text {old }}=P_{1}$. If $\Delta P=P_{\text {new }}-P_{\text {old }}<0$, the yaw direction of wind turbine is considered to be correct; If $|\Delta P|>P_{e 2}$, the yaw movement after haven't meet the requirements of system setting, also need to continue to drift action, by the method of recursive calls to detect the output power of wind turbines, until after $|\Delta P|<P_{e 1}$ and $|\Delta P|<P_{e 2}$, argues that the yaw motion to set target system, stop the yaw motion, at the same time at the moment the latest wind turbine power output value $P_{\text {now }}$ to $P_{\text {min }}$, more value for the next time the yaw motion. If $\Delta P=P_{\text {new }}-P_{\text {old }}>0$ appears during yaw action, the yaw direction is wrong.
After returning to the initial position, change the yaw direction.

When the wind speed changes, it will have an intuitive impact on the power captured by the wind turbine. The direct manifestation is that the output power of the wind turbine generator changes significantly. After the wind speed and wind direction are changed, according to the previous description, if the condition $\left|P_{\text {now }}-P_{\text {min }}\right|>P_{e 1}$ is met, the wind turbine yaw system will start and rotate according to the pre-set direction. After the rotation, it will be judged whether $\Delta P=P_{\text {now }}-P_{\text {old }}<0$ is established, if it is established, the next step is determined, if $\Delta P<0$ and $|\Delta P|<P_{e 2}$ then Satisfy the yaw requirements, stop the yaw action, if $\Delta P<0$ is not established, perform the reverse yaw, and again determine whether the yaw accuracy is satisfied, until it is satisfied.

Among them, $P_{e 1}$ determines the frequency of turning on the yaw motor, and $P_{e 2}$ this parameter will affect the control effect of the yaw system. If the setting value of $P_{e 1}$ is larger, the control system is less sensitive to power changes. If the setting value of $P_{e 2}$ is small, near the minimum power value, the yaw motor will rotate forward and backward after starting, reducing the control effect of the system.

The block diagram of Hill Climbing Control algorithm for yaw controller is shown in Figure 2. In the figure, $P_{\mathrm{s}}$ is the cumulative power value. $k$ is the current accumulated power number; $m$ is the power accumulation number set by the system. 


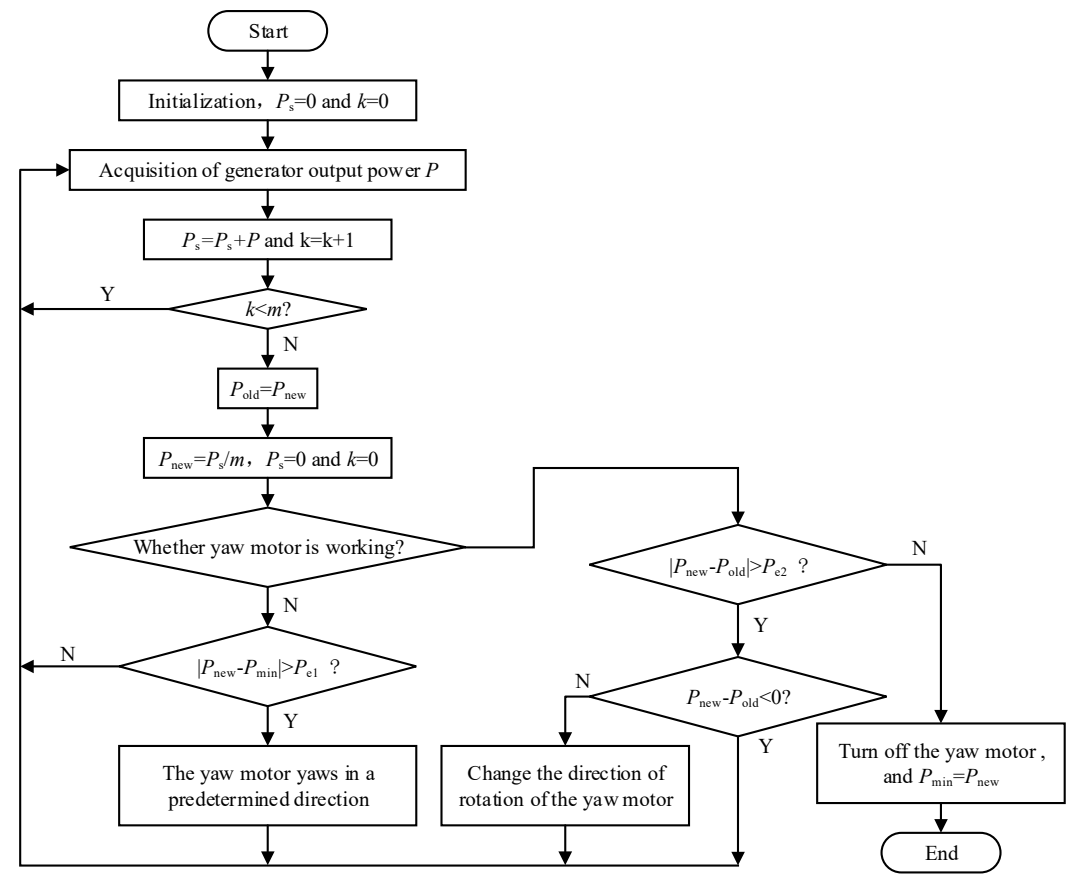

Figure 2. Flow chart of Hill Climbing Control for yaw system

\section{Maximum power calculation of yaw system under typhoon condition}

\subsection{Calculation of maximum typhoon wind speed}

Under typhoon conditions, the maximum wind speed of the typhoon needs to be calculated, and the typhoon wind field model is obtained based on the basic theory of the relationship between the atmospheric pressure field and the wind speed. This paper adopts the wind field model proposed by Batts, which is a relatively mature and simpler wind field model. The model includes the sea surface typhoon wind field model and the attenuation model considering the typhoon registration.

In this model, the maximum gradient wind speed $V_{g x}$ is:

$$
V_{g x}=K \sqrt{\Delta P}-\left(R_{\max } / 2\right) f
$$

where $K$ is the empirical constant, taking $6.72 ; R_{\max }$ is the maximum wind speed radius $(\mathrm{km}) ; \Delta P$ is the typhoon center pressure difference $(\mathrm{hPa}) ; f$ is the Coriolis coefficient, and its expression is as follows:

$$
f=2 \Omega \sin \psi
$$

where $\Omega$ is the rotation speed of the earth $(\operatorname{rad} / \mathrm{s}) ; \psi$ is the latitude of the air cluster.

At the height of $h_{\mathrm{M}}$ above the sea surface, the average maximum wind speed within $10 \mathrm{~min}$ is $V_{h, R_{\max }}$ :

$$
V_{h, R_{\max }}=0.865 V_{g x}+0.5 V_{T}
$$

where $V_{T}$ is the moving speed of typhoon.

After a typhoon makes landfall, friction will cause a certain wind speed attenuation. The ratio of wind speed $V^{t}(h)$ (at height $h_{\mathrm{m} \text {, average within } 10 \text { minutes) after }}$ landing to wind speed $V^{w}(h)$ (at height $h_{\mathrm{m}}$, average within 10 minutes) on the sea surface is:

$$
\frac{V^{t}(h)}{V^{w}(h)}=\frac{1}{0.2 p \ln \frac{h}{Z_{0}}}
$$

where $p$ is the obstacle factor; $Z_{0}$ is the rough length. For open coastal areas, the value of $p$ is 0.85 ; the value of $Z_{0}$ is 0.005 . $^{t}(h)$ is the maximum wind speed at $h \mathrm{~m}$ in typhoon condition.

\subsection{Maximum power measurement for yaw system}

According to the operating principle of the wind turbine yaw system, it can be seen that the operation of the wind turbine yaw system is mainly affected by the following forces: $M_{z}$ is the brake damping moment applied by the brake damper to prevent the fluctuation of yaw system during operation, and it shall not be considered when the yaw motor is in motion state; $M_{f}$ is the frictional resistance moment on the slewing support device. $M_{w}$ is the rotational resistance torque caused by the wind pressure acting on the nacelle. Since the yaw speed is relatively low, the yaw inertia torque is temporarily not considered.

To simplify the model, it is necessary to convert the resistance moment of nacelle into the motor shaft. For yaw system of wind turbine, yaw resistance moment is: 


$$
M=M_{w}+M_{f}+M_{z}
$$

Here, the frictional resistance torque ${ }^{M_{f}}$ can be calculated by the following formula:

$$
M_{f}=N_{m} \times \mu \frac{D_{0}}{2}
$$

where $N_{m}$ is the total pressure on the yaw bearing, which is composed of the pressure formed by the mass of the wind turbine and the mass of the nacelle; $D_{0}$ is the diameter of yaw bearing; ${ }^{\mu}$ is the friction coefficient of yaw bearing.

The calculation formula of rotary resistance moment $M_{w}$ is as follows:

$$
M_{w}=F_{\max } \times \frac{R_{0}}{2}
$$

where $R_{0}$ is the length of the wind turbine blade; $F_{\text {max }}$ is the maximum wind pressure suffered by the engine room. Its calculation formula is as follows:

$$
F_{\max }=\frac{1}{2} \rho V_{\max }^{2} C_{t} A_{b} B
$$

where $\rho$ is the air density $\left(1.225 \mathrm{~kg} / \mathrm{m}^{3}\right)$; $V_{\max }$ is the maximum wind speed; $C_{t}$ is the resistance coefficient, take $1.7 ;^{A}$ is the projected area of the blade; $B$ is the number of blades.

Then the resistance moment $T_{m}$ converted to the motor shaft is:

$$
T_{m}=\frac{M}{4 i_{1} i_{2} \eta}
$$

where $\eta$ is the transmission efficiency from engine room to yaw motor shaft, taking $80 \%$. follows:

The angular velocity $\omega_{m}$ of the motor is calculated as

$$
\omega_{m}=i_{1} i_{2} \omega_{r}
$$

where $i_{1}, i_{2}$ are yaw gear box transmission ratio and small big gear transmission ratio respectively; $\omega_{r}$ is the yaw angular velocity, which is generally set as $0.5^{\circ} \mathrm{s}$.

From the product of motor torque and angular velocity, the power of yaw motor can be obtained as follows:

$$
P_{m}=T_{m} \times \omega_{m}=\frac{M}{4 i_{1} i_{2} \eta} \times i_{1} i_{2} \omega_{r}=\frac{M \omega_{r}}{4 \eta}
$$

where $P_{m}$ is the actual power consumed by the calculated single yaw motor.

\section{Configuration and selection of diesel generator}

Under typhoon conditions, it is assumed that the rated selfuse electric power of a single wind generator set is $P_{0}$, the power of yaw motor is $P_{1}$, rate of yaw motor is $K_{1}$ ( $K_{1}$ is 1.0 if the working rate of yaw system of full-court fan is $100 \%$ )..At this time, the power ratio of yaw motor of a single wind turbine generator set is $P_{1} / P_{0}$, and the ratio ratio is $P_{1} K_{1} / P_{0}$. Then the simultaneous rate of active power of a single wind field is $I_{k}$ :

$$
I_{k}=\frac{P_{1} K_{1}}{P_{0}}(k=1,2, \cdots N)
$$

where $N$ means that there are $N$ wind turbines in the whole wind farm, and the power consumed by the whole wind farm can be expressed as:

$$
P=\sum_{k=1}^{N} I_{k} \cdot P_{0}
$$

According to the actual power consumed by the whole wind farm, the optimal configuration and selection of diesel generator set can be carried out.

\section{Case study}

Putuo 6 Offshore Wind Farm located in the southeast of Liuheng Island, Zhoushan City, Zhejiang Province. The Wind farm is equipped with 63 wind turbines of 4.0MW. Putuo 6 Offshore wind Farm is located in the typhoonprone sea area, with the maximum wind speed of $49.6 \mathrm{~m} / \mathrm{s}$ and maximum wind speed of $64.48 \mathrm{~m} / \mathrm{s}$ at a height of $90 \mathrm{~m}$ within 50 years. Therefore, Siemens SWT4.0-130 units are selected. Siemens SWT4.0-130 is a three-blade wind turbine with 6 yaw motors per unit. The specific parameters are as follows: the mass of the wind turbine is $120 \mathrm{~T}$, the mass of the engine room is $167 \mathrm{~T}$, the diameter of the yaw bearing is $3.035 \mathrm{~m}$, the friction coefficient of the yaw bearing is 0.08 , the transmission ratio of the yaw gear box is 975 , the transmission ratio of small and large gears is $154 / 15$, and the rated power of the yaw motor is $5.5 \mathrm{~kW}$. The impeller has a diameter of 130 meters, a blade length of $63.5 \mathrm{~m}$, and a maximum chord length of $3 \mathrm{~m}$.

Take the maximum wind speed of $49.6 \mathrm{~m} / \mathrm{s}$ as an example, and the air density is $1.225 \mathrm{~kg} / \mathrm{m}^{3}$. The projected area of a single blade is $97.5 \mathrm{~m}^{2}$. The maximum wind pressure experienced by the nacelle of a three-blade wind turbine is $7.49 \times 10^{5} \mathrm{~N}$, and the magnitude of the rotational resistance moment caused by the wind pressure acting on the nacelle is $2.38 \times 10^{7} N \cdot M$, and friction resistance moment on slewing support device is $3.41 \times 10^{5} N \cdot M$. Therefore, the yaw resistance torque of a single wind turbine is $2.41 \times 10^{7} \mathrm{~N} \cdot \mathrm{M}$. The value of the resistance torque converted to the motor shaft is 
$752.37 N \cdot M$. The angular velocity of the motor is $87.35 \mathrm{rad} / \mathrm{s}$. Then the actual power of the yaw system of a single wind turbine is about $65.71 \mathrm{KW}$. When the yaw system of the entire wind farm works at $100 \%$, the total power consumption of the yaw system of 63 wind turbines is $4139.73 \mathrm{KW}$.

\section{Conclusion}

Wind energy as a renewable energy is more and more favored by countries all over the world. Therefore, it is timely to pay attention to and make efforts to promote the rapid development of wind power generation technology in China, and its social and economic benefits are selfevident. In this paper, the working mode of yaw control system under typhoon condition is optimized. By calculating the power of a single yaw motor, the maximum power of the yaw system is calculated. On this basis, the standby diesel generator set is selected and its capacity configuration is optimized, so that it can meet the requirements of the yaw system of the whole wind farm.

\section{References}

1. Wang Z, Zhao Y, Li F , et al. Extreme Dynamic Responses of MW-Level Wind Turbine Tower in the Strong Typhoon Considering Wind-Rain Loads[J]. Mathematical Problems in Engineering,2013,(20135-16), 2013, 2013:133-174.

2. Arana I, Hernandez A, Thumm G, et al. Energization of Wind Turbine Transformers With an Auxiliary Generator in a Large Offshore Wind Farm During Islanded Operation[J]. IEEE Transactions on Power Delivery, 2011, 26(4):2792-2800.

3. Q. Wang, Z. Yu, R. Ye, Z. Lin and Y. Tang, "An Ordered Curtailment Strategy for Offshore Wind Power Under Extreme Weather Conditions Considering the Resilience of the Grid," in IEEE Access, vol. 7, pp. 54824-54833, 2019, doi: 10.1109/ACCESS.2019.2911702.

4. Zhang X, Wang M, Wang Y, et al. Research on Failure of Offshore Wind Turbine Tower in Typhoon[C]// 2018 OCEANS - MTS/IEEE Kobe Techno-Ocean (OTO). IEEE, 2018.

5. Ma Z, Li W, Ren N, et al. The typhoon effect on the aerodynamic performance of a floating offshore wind turbine $[\mathrm{J}]$. Journal of Ocean Engineering and Science, 2017, 2( 4):279-287. 